\title{
Presentación
}

\section{La Formación Inicial del Profesorado para una Educación Inclusiva: Desafíos, Oportunidades y Transformaciones}

\author{
Constanza Herrera-Seda * \\ Universidad de Santiago de Chile, Chile
}

El movimiento por una educación inclusiva, que ha tomado fuerza en las últimas décadas, ha destacado el rol del profesorado en la transformación de las aulas, las instituciones y el sistema educativo en su conjunto. Más allá de este reconocimiento, los docentes en ejercicio suelen experimentar dificultades para liderar procesos de enseñanza y aprendizaje con un foco inclusivo, lo que ha llevado a reconocer la importancia de la formación inicial de profesores y a poner en cuestionamiento la pertinencia de los actuales modelos y programas de formación para responder a este fenómeno.

En el presente número de la revista se aborda el desafío de la formación inicial de profesores para una educación inclusiva, a partir de tres interrogantes clave ¿Qué demanda el sistema escolar a la formación inicial del profesorado en materia de inclusión? ¿Cuán preparadas están las universidades para desarrollar procesos de formación con un sello inclusivo? ¿Qué condiciones debería tener la formación inicial del profesorado para garantizar el desarrollo de las competencias necesarias para ejercer el rol docente desde un enfoque inclusivo?

Las contribuciones a este número permiten dar respuesta a estas interrogantes a partir tres ideas centrales sobre la formación inicial de profesores, en las que vale la pena detenerse si resulta de interés comprender la complejidad del proceso y tomar decisiones acertadas en relación a su fortalecimiento.

\section{Las escuelas se están transformando con un sentido inclusivo y sus experiencias son claves para orientar la formación inicial de profesores}

Hoy en día las escuelas atraviesan un proceso de profunda transformación, entre otros factores, a partir de los cambios en las políticas educativas, en las que el eje de inclusión se ha constituido un núcleo fundamental. Al respecto, el trabajo desarrollado por Dominique Manghi, Camila Saavedra y Nicoley Bascuñan "Prácticas educativas en contextos de educación pública, inclusión más allá de las contradicciones”, da cuenta de los cambios que han venido sucediendo en el contexto escolar, a partir de la instalación de nuevos marcos legales y de acción que emanan de dichas políticas. Este mismo trabajo pone de manifiesto las contradicciones a las que se enfrentan las instituciones educativas y sus actores en el proceso de tránsito desde una respuesta asistencialidad a la diversidad del estudiantado, hacia otra que se sustenta en los principios valóricos y políticos del enfoque inclusivo.

*Contacto: constanza.herrera@gmail.com 
A partir de estas transformaciones en las políticas educativas y de los acelerados cambios sociales, las escuelas se han visto ante el reto que supone el proceso de diversificación de la matrícula. Tal como nos muestra el trabajo de Rodolfo Cruz "Inclusión, discapacidad y profesores: algunas reflexiones para repensar las prácticas (político-educativas)", la presencia de estudiantes tradicionalmente excluidos del sistema educativo, como son las personas en condición de discapacidad, ha comenzado a trastocar el orden tradicionalmente establecido en las escuelas, así como a interpelar las concepciones de los docentes, abriendo una oportunidad para movilizar a las instituciones escolares en la construcción de nuevos sentidos desde la inclusión y cambiar los modos en que hasta ahora se han conducido los procesos de enseñanza y aprendizaje.

En relación a la inclusión, el proceso de cambio en las escuelas refleja la complejidad de los fenómenos educativos y se desarrolla a partir de avances a un ritmo lento. Al respecto, el trabajo de Mirtha Jara y Pilar Jara "Concepciones y prácticas evaluativas declaradas por los docentes en respuesta a las necesidades educativas especiales de carácter permanente", da cuenta de la persistencia de creencias y prácticas tradicionales en torno a la diversidad y su abordaje. En el ámbito de la evaluación de los aprendizajes, las autoras ponen de relieve la toma de conciencia por parte del profesorado sobre las particularidades de los estudiantes, sin embargo, observan que estos continúan respondiendo a la diversidad a través de adecuaciones curriculares y no desde la implementación de propuestas evaluativas flexibles, que garanticen el acceso y participación en el aprendizaje de todo el alumnado.

Ahora bien, las tensiones que emanan de la persistencia de estos paradigmas en competencia, pueden ser consideradas como oportunidades para que los actores educativos reflexionen acerca de la calidad y equidad de la formación. El trabajo de María Virginia Hael "Abordajes de la lectura por parte de sordos en contextos bilingües en países de habla hispana” constituye un buen ejemplo de este fenómeno. Ya que, si bien aborda las condiciones de exclusión de un grupo de estudiantes particular, cuyas características y requerimientos no podríamos obviar, sus resultados nos permiten una discusión amplia acerca de la enseñanza y el aprendizaje del lenguaje en contextos de aulas heterogéneas.

Las transformaciones que están ocurriendo en las instituciones escolares, llaman a anhelar una formación inicial del profesorado con foco inclusivo, que permita a los futuros docentes desenvolverse en contextos profesionales híbridos, donde persisten prácticas tradicionales de integración escolar y, al mismo tiempo, emergen nuevos sentidos y actuaciones coherentes con un enfoque inclusivo. En consecuencia, se requiere que el profesorado en formación tenga acceso tempranamente a la realidad del aula, a partir de experiencias prácticas que no solo permitan conocer dicho contexto, sino además reflexionar acerca de este y comprometerse con una perspectiva inclusiva que, más allá del discurso, esté instalada en las prácticas cotidianas del ejercicio de la profesión.

\section{La universidad está siendo tensionada por la diversidad de sus estudiantes y avanzar con sentido inclusivo resulta relevante, especialmente, para la formación inicial de profesores}

La ampliación de los mecanismos de acceso a la educación superior, no solo ha aumentado la matrícula universitaria, sino que también ha llevado a que estas instituciones educativas se enfrenten al reto de formar estudiantes diversos y a grupos de la población que, hasta ahora, se encontraban escasamente representados en la universidad. 
El trabajo de Juan Carlos Ocampo "Discapacidad, inclusión y educación superior en Ecuador: El caso de la Universidad Católica de Santiago de Guayaquil” da cuenta del fenómeno recién descrito y muestra que, a pesar del interés de las instituciones, académicos y profesionales, el acceso a la universidad de estudiantes tradicionalmente excluidos es aun restrictivo, de modo que grupos importantes de la población siguen estando menos representados en la formación en este nivel. A lo anterior, habría que agregar, por cierto, la importancia de generar condiciones para que todo el estudiantado pueda progresar en la formación profesional.

Una dimensión fundamental asociada a este último punto, es tratada en el trabajo de Eddy Paz "Competencias del profesorado universitario para la atención a la diversidad en la educación superior", que pone de manifiesto la importancia de contar con docentes universitarios que favorezcan la participación, el pluralismo y la democratización de los espacios educativos. En este trabajo se enfatiza el compromiso que deben asumir las universidades, no solo promoviendo la presencia de estudiantes diversos, sino también implementando procesos de enseñanza y aprendizaje ajustados a dicha heterogeneidad, donde las competencias del cuerpo docente constituyen un eje fundamental de trabajo.

El desafío de la inclusión en el contexto universitario resulta especialmente relevante cuando se aborda la formación inicial del profesorado. Por una parte, las propuestas formativas deberían garantizar que todos los futuros docentes desarrollen las múltiples competencias requeridas para el ejercicio de la profesión. Por otro, deberían propiciar situaciones de aprendizaje que les permitan experimentar climas de aula inclusivos y democráticos, que en la mayoría de los casos no han tenido oportunidad de vivenciar en su formación escolar, y que tienen la potencialidad de constituirse en referentes fundamentales para que la futura práctica docente se desarrolle desde un enfoque inclusivo.

\section{La formación inicial de profesores para una educación inclusiva debería transformarse profundamente y desde la raíz}

Considerando la relevancia de la formación inicial del profesorado en el desarrollo de la identidad profesional y de las competencias necesarias para el ejercicio docente en el contexto escolar, transformar las trayectorias de formación con un sentido inclusivo resulta una tarea fundamental, que debería permitir repensar la naturaleza de los procesos de formación y los principios sobre los cuales se sostiene, es decir, las concepciones acerca del aprendizaje, la enseñanza, la diversidad, el rol docente, entre otros.

El trabajo de Pablo Castillo y Carlos Miranda "Actitud hacia la inclusión de los estudiantes de pedagogía de una universidad estatal chilena" sustenta la importancia de conducir procesos de profundo cambio y fortalecimiento de la formación inicial desde un enfoque inclusivo. Al respecto, los autores señalan la relevancia de que los profesores en formación no solo desarrollen una actitud positiva hacia la inclusión, sino que también se sientan preparados para contribuir a esta en el ejercicio de su profesión, desarrollando prácticas pedagógicas acorde a los principios de este enfoque.

En consecuencia, los programas de formación de profesores están llamados a discutir, desde un enfoque inclusivo, sus principios orientadores, rescatando aquellos valores, dispositivos y prácticas que promueven una educación inclusiva, e identificando los que, por el contrario, se constituyen en barreras para que los futuros profesores se sientan convocados e implicados con el aprendizaje y la participación de todo el estudiantado, y 
posean los conocimientos y habilidades necesarias para responder a su amplia diversidad. La reflexión continua sobre este punto constituye una valiosa oportunidad de transformación profunda y sustentable en las instituciones universitarias.

Por otro lado, la formación de profesores requiere necesariamente cambiar desde la raíz, es decir, movilizar dicha transformación desde los docentes formadores de profesores. En este sentido, el trabajo que hemos elaborado en conjunto con Carolina García y Carlos Vanegas "Competencias docentes para una pedagogía inclusiva. Consideraciones a partir de la experiencia con formadores de profesores chilenos" pone de relieve que los docentes vinculados a la formación inicial del profesorado suelen desarrollar prácticas que contribuyen a la construcción de ambientes de aprendizaje inclusivos en la universidad, aun cuando se requiere seguir fortaleciendo sus competencias para innovar en el aula y diseñar situaciones de aprendizaje flexibles acordes a la diversidad del alumnado.

Al mismo tiempo, en este trabajo se discute la importancia de definir cuáles son las competencias que requieren los formadores para contribuir de manera efectiva a que los futuros profesores se comprometan con una perspectiva inclusiva, desarrollen las habilidades necesarias para diseñar experiencias de aprendizaje para todos y se transformen en agentes movilizadores de cambio en las instituciones escolares. Por tanto, una perspectiva inclusiva de la formación inicial de profesores emplaza a los docentes y actores universitarios a contribuir a que los docentes en formación puedan desarrollar la dimensión profesional, ética y política que implica el asumir el rol de profesor desde un enfoque pedagógico inclusivo, que apuesta por el aprendizaje de todos, sin exclusiones.

Finalmente, resulta necesario resaltar que, al igual que en el contexto escolar, alcanzar una educación inclusiva en el contexto universitario y particularmente en la formación de profesores corresponde a un desafío para el cual no existe un único modelo o ruta de transformación. En cada caso dependerá, entre otras cosas, de las políticas nacionales en materia de inclusión y docencia, de los valores que movilizan la cultura de cada universidad, de las particularidades de las propuestas formativas de las distintas instituciones, así como de los recursos y las barreras a las que se enfrentan los actores de cada programa. Es de esperar entonces, que este número permita a los lectores y a quienes participan de la formación docente, identificar las cuestiones que les desafían particularmente, así como pensar modos innovadores de abordar la formación de profesores para una educación inclusiva. 